pregnancy. $29.3 \%$ of pregnant women both smoked and drank before pregnancy reducing to $15.1 \%$ during pregnancy. Adjusted analysis showed that birth weight was negatively associated with continual smoking, $-231 \mathrm{~g}$ ( $95 \%$ CI -318.3 to -144.5$)$, whilst quitting or decreasing smoking was associated with $-68.5 \mathrm{~g}$ reduction ( $95 \%$ CI -119.7 to -17.4 ) compared to nonsmokers. No association with birth weight was observed for alcohol consumption $(-0.16 \mathrm{~g}, 95 \% \mathrm{CI}-69.9$ to 69.6$)$.

Conclusions Timing of quit smoking interventions should concentrate on the pre-pregnancy period to ensure optimal benefits on birth weight. These results suggest that smoking cessation advice provided in the ante natal period may have limited benefit.

\section{P14 INTERVENTIONS TO INCREASE THE EARLY INITIATION OF ANTENATAL CARE IN SOCIALLY DISADVANTAGED AND VULNERABLE WOMEN: A SYSTEMATIC REVIEW}

doi:10.1136/jech.2010.120477.14

L Oakley, R Gray, J J Kurinczuk, P Brocklehurst, J Hollowell. National Perinatal Epidemiology Unit, University of Oxford, Oxford, UK

Objective To systematically evaluate the effectiveness of interventions to increase the early initiation of comprehensive antenatal care in socially disadvantaged and vulnerable women.

Design Systematic review.

Data Sources Major bibliographic databases (Medline, Cinahl, Embase, PsycINFO, HMIC, CENTRAL) and other online libraries and resources were searched to identify relevant English language journal articles published 1990-2009. We included comparative studies (experimental or observational) evaluating the effectiveness of an intervention on the proportion of women initiating antenatal care by any defined cut-off point $\leq 20$ weeks in a disadvantaged or vulnerable population. In order to focus on interventions relevant in the context of the UK National Health Service, we excluded studies from low-income countries and those relating to financial interventions such as extension of health insurance coverage or similar. Review Methods Two reviewers independently extracted data for eligible studies; assessed internal validity using the GATE checklist and considered whether the studies provided evidence of a beneficial effect.

Results Over 3000 citations were screened. Sixteen eligible studies were identified; 14 conducted in the USA, one in Australia and one in the UK. All were observational evaluations. Twelve studies evaluated interventions targeted at specific disadvantaged or vulnerable subgroups of the population (predominantly ethnic minority women or teenagers); the remaining studies evaluated interventions in more generally socioeconomically disadvantaged populations. Eleven studies evaluated interventions that involved outreach or other community-based services, and five evaluated interventions that involved alternative models of clinic-based antenatal care. Overall, the quality of evidence was poor. Only one study, which evaluated a US home visiting intervention delivered by paraprofessional women to pregnant adolescents, was considered to have adequate internal validity. The reviewers considered the evidence relating to this intervention to be inconclusive but consistent with a possible beneficial effect of the intervention on timing of initiation of antenatal care.

Conclusion We found insufficient evidence of adequate quality to reliably conclude that any of the interventions considered were effective at increasing the early initiation of antenatal care in socially disadvantaged and vulnerable women. There was weak evidence of effectiveness relating to one intervention based on home visiting for pregnant adolescents. Findings were inconclusive for all other included interventions, although we identified several strategies that might warrant further consideration and possibly more robust evaluation. The results of this review highlight the paucity of evidence and the need for further high quality research to ensure that future service innovations are evidence based.

\section{P15 EFFECT OF TRAINING DOCTORS IN COMMUNICATION SKILLS ON SYRIAN WOMEN'S SATISFACTION DURING LABOUR: A STEPPED WEDGE CLUSTER RANDOMISE TRIAL}

doi:10.1136/jech.2010.120477.15

${ }^{1} \mathrm{M}$ Kanaan, ${ }^{2} \mathrm{H}$ Bashour, ${ }^{2} \mathrm{~A}$ Abdulsalam, ${ }^{2} \mathrm{M}$ Kharouf, ${ }^{2} \mathrm{~S}$ Cheikha, ${ }^{2} \mathrm{M}$ Tabbaa. ${ }^{1}$ Department of Health Sciences, University of York, York, UK; ${ }^{2}$ Faculty of Medicine, Damascus University, Damascus, Syria

Objective To test the effect of training residents in interpersonal and communication skills on women's satisfaction with patient-doctor relationship in labour and delivery rooms.

Design A stepped-wedge cluster randomised trial.

Setting Maternity wards in four teaching hospitals in Damascus and homes of participating women.

Participants Women delivering in the hospitals under study, via vaginal delivery with living baby, who consent to participate in the study. Difficult labour and high-risk pregnancies are excluded. Residents working in the study hospitals during the implementation phase who agree to take part in the study.

Main Outcome Measure Women's satisfaction with interpersonal relationships in labour and delivery rooms measured via a series of questions on a Likert scale and based on the Medical Interview Satisfaction Scale.

Results Women were on average 25 years old, 95\% were homemakers, $76 \%$ had primary education, $48 \%$ lived in shared accommodation and $26 \%$ were nulliparous. At the individual level, the mean score of overall satisfaction of women was $68.66(\mathrm{SD}=14.24)$ out of a possible score of a 100 in the control group and 70.79 $(\mathrm{SD}=13)$ in the treatment group. At the hospital level, the mean scores of overall satisfaction of women were $70(\mathrm{SD}=4.70)$ and 70.99 $(\mathrm{SD}=4.85)$ for the control and treatment group, respectively. Using (generalised) linear mixed models approach to account for the study design, we were not able to detect a difference between the treatment and control group on the overall satisfaction of women.

Conclusion The training package does not seem to be associated with higher overall satisfaction scores.

\section{P16 QUANTIFYING THE RISK OF DEPRIVATION ON PRETERM BIRTH IN UK MATERNITY UNITS}

doi:10.1136/jech.2010.120477.16

${ }^{1} \mathrm{D}$ Taylor-Robinson, ${ }^{2} \mathrm{U}$ Agarwal, ${ }^{1} \mathrm{M} \mathrm{J}$ Platt, ${ }^{3} \mathrm{P}$ Diggle, ${ }^{4} \mathrm{~B}$ Yoxall, ${ }^{5} \mathrm{Z}$ Alfirevic. ${ }^{1}$ University of Liverpool, Liverpool, UK; ${ }^{2}$ Liverpool Women's NHS Foundation Trust, Liverpool, UK; ${ }^{3}$ CHICAS, Lancaster University, Lancaster, UK; ${ }^{4}$ Women's NHS Foundation Trust, Liverpool, UK; ${ }^{5}$ Fetal and Maternal Medicine, University of Liverpool, Liverpool, UK

Objective To explore risk factors for preterm birth (PTB) between 24 +0 and $34+0$ gestational weeks in the UK's largest maternity unit, with a particular focus on low risk pregnancies and the effect of socioeconomic status.

Design Retrospective cohort study of routinely collected obstetric and neonatal data.

Setting 50486 singleton pregnancies booked at the Liverpool Women's NHS Foundation Trust for all women delivering after 24 +0 weeks gestation over a 7-year period from 2002 to to 2008 .

Main Outcome Measure The primary outcome was preterm birth. Pregnancies were stratified into three groups: low risk; those complicated by medical problems; pregnancies in women with a 